\title{
Plasma Exosomal Long Non-Coding RNAs Serve as Biomarkers for Early Detection of Colorectal Cancer
}

\author{
Dongzhi Hu Yang Zhan Kegan Zhu Ming Bai Jiayi Han Yiran Si \\ Haiyang Zhang Dalu Kong \\ Tianjin Medical University Cancer Institute and Hospital, National Clinical Research Center for Cancer, \\ Tianjin's Clinical Research Center for Cancer, Key Laboratory of Cancer Prevention and Therapy, Tianjin \\ Medical University, Tianjin, China
}

\section{Key Words}

Circulatory exosomes $\bullet$ Long non-coding RNA • Biomarker • Colorectal cancer • Diagnosis

\begin{abstract}
Background/Aims: Colorectal cancer (CRC) is the third most commonly diagnosed malignancy and the second leading cause of cancer-related deaths worldwide. Thus, methods for early diagnosis of CRC are urgently needed. We aimed to identify potential long non-coding RNAs (IncRNAs) in circulatory exosomes that may serve as biomarkers for the detection of early-stage CRC. Methods: Exosomes from the plasma of CRC patients $(n=50)$ and healthy individuals $(n=$ 50) were isolated by ultracentrifugation, followed by extraction of total exosomal RNAs using TRIzol reagent. Microarray analysis was used for exosomal IncRNA profiling in the two groups, and real-time quantitative reverse transcription-polymerase chain reaction (qRT-PCR) was used to determine the expression level of IncRNAs in all patients and healthy subjects. Results: The expression of six IncRNAs (LNCV6_116109, LNCV6_98390, LNCV6_38772, LNCV_108266, LNCV6_84003, and LNCV6_98602) was found to be significantly up-regulated in CRC patients compared with that in healthy individuals by qRT-PCR. The receiver operating characteristic curve was used to verify their diagnostic accuracy. The values of the area under the curve for these IncRNAs were 0.770 (LNCV6_116109), 0.7500 (LNCV6_98390), 0.6500 (LNCV6_38772), 0.6900 (LNCV_108266), 0.7500 (LNCV6_84003), and 0.7200 (LNCV6_98602). Conclusion: Our study suggested that the expression of these six exosomal IncRNAs (LNCV6_116109, LNCV6_98390, LNCV6_38772, LNCV_108266, LNCV6_84003, and LNCV6_98602) was significantly up-regulated in the plasma of CRC patients, and that they may serve as potential non-invasive biomarkers for early diagnosis of CRC.
\end{abstract}

Dalu Kong

and Haiyang Zhang
Tianjin Medical University Cancer Institute and Hospital; Huan hu xi Road 18, Tianjin 300060 (China) Tel. +86022233401230150, Fax +8602223537796

E-Mail kongdl@hotmail.com; zhanghaiyang@tmu.edu.cn

\section{KARGER}




\section{Cellular Physiology Cell Physiol Biochem 2018;51:2704-2715 \begin{tabular}{ll|l} 
DOl: 10.1159/000495961 & $\begin{array}{l}\text { O 2018 The Author(s). Published by S. Karger AG, Basel } \\
\text { www.karger.com/cpb }\end{array}$ \\
\cline { 2 - 3 }
\end{tabular} \\ Hu et al.: Exosome IncRNAs are Biomarkers for CRC}

\section{Introduction}

Colorectal cancer (CRC) is one of the three most common malignancies in the world [1]. Although the mortality rate has declined due to improvements in treatment [2], early diagnosis of CRC is still a major challenge. Current screening methods, such as fecal occult blood test (FOBT), fecal immunochemical test, and colonoscopy have various limitations, with only 30-40\% of patients being detected at an early stage [3]. Therefore, the development of a new non-invasive approach to improve the early detection of CRC is urgently needed.

Exosomes are 30-100 nm cell-derived vesicles containing a wide variety of molecules formed by endocytosis, and can be found in several body fluids (such as blood, urine, saliva, pleural fluid, and amniotic fluid) [4, 5]. Recent research has shown that exosomes are stable in blood [6] and can deliver a large cargo of proteins, lipids, and nucleic acids, including long non-coding RNA (lncRNAs) [7, 8]. Humoral exosomes are considered to be promising biomarkers for cancer [9], but the potential role of plasma exosomes in the diagnosis of CRC has not yet been reported.

lncRNAs are a novel class of non-coding RNAs defined as non-protein RNA transcripts greater than 200 nucleotides in length [10]. The process of transcription and modification of IncRNAs includes RNA polymerase II transcription, 5'-capping, poly-adenylation, and alternative splicing, which is similar to that of protein-coding genes [11]. Over 20 years ago, with the discovery of the paternally imprinted maternally expressed transcript (H19) gene, scientists realized that not all long RNA transcripts are mRNAs [12]. Since then, over 13, 000 lncRNA genes that produce over 23, 000 lncRNAs in humans have been discovered, and the number is rising rapidly [13]. Although lncRNAs represent a large family, their specific mechanisms of action have not yet been thoroughly elucidated. Previous studies have demonstrated that IncRNAs play a diverse role in biological processes. Firstly, lncRNAs interact with chromatin-modifying proteins or transcription factors through their specific protein-binding motifs. Moreover, IncRNAs can directly bind to a specific DNA sequence to form an RNA-DNA triplex structure that can block the transcription process [14]. The process of IncRNA transcription may also provide an "open" chromatin domain under certain circumstances, which then triggers the expression of nearby genes [15]. Lastly, IncRNAs can also silence the nearby genes by occupying the transcription factor binding sites [16]. It has been shown that lncRNAs are involved in almost every step of the life cycle [17] and play an increasingly important role in cancer. Several studies have revealed the function of IncRNAs in the tumorigenesis, progression, and diagnosis of various cancers, such as prostate, bladder, kidney [18], breast [19], and lung cancer [20]. Recent studies have suggested that extracellular-vesicle-linked lncRNAs can foster dictated patterns of transregulation in recipient cells, and are involved in envisaging novel mechanisms of epigenetic regulation, cellular reprogramming, and genomic instability elicited in target cells, leading to the generation of senescence-associated tumor-initiating cells, which are resistant to chemotherapy [21]. Additionally, several researchers have demonstrated their potential role in the diagnosis, prognosis, and treatment of CRC [22, 23]. However, the role of exosomal lncRNAs in the early diagnosis of CRC remains unclear.

In this study, we compared a number of IncRNAs in the plasma exosome samples between CRC patients and control individuals by RNA sequencing, and then expanded the sample capacity to test its feasibility. Our data suggested that namely, LNCV6_116109, LNCV6_98390, LNCV6_38772, LNCV_108266, LNCV6_84003, and LNCV6_98602, might be considered as novel biomarkers for diagnosis of early-stage CRC. 


\section{Cellular Physiology Cell Physiol Biochem 2018;51:2704-2715 and Biochemistry Publisnecontune: \begin{tabular}{l|l} 
DOI: 10.1159/000495961 2018 The Author(s). Published by S. Karger AG, Basel \\
www.karger.com/cpb
\end{tabular}}

Hu et al.: Exosome IncRNAs are Biomarkers for CRC

\section{Materials and Methods}

Patients and clinical specimens

For this study, whole blood samples were collected from CRC patients at Tianjin Medical University Cancer Institute and Hospital. None of the experimental subjects underwent prior colorectal surgery or neoadjuvant radiotherapy/chemotherapy. Fresh plasma samples $(3 \mathrm{~mL})$ were collected in ethylenediamine tetraacetic acid tubes from each of the subjects. These samples were centrifuged at $3000 \times g$ for $10 \mathrm{~min}$ at $4^{\circ} \mathrm{C}$ and then stored at $-80^{\circ} \mathrm{C}$. This study was approved by the Ethics Committee of Tianjin Medical University Cancer Institute and Hospital. Written informed consent was obtained from all participants.

\section{Plasma exosome isolation}

Differential centrifugation is considered a gold standard and is the most common method to isolate exosomes [24]. Firstly, the samples were centrifuged twice at 3, $000 \times g$ and 10, $000 \times g$ for $20 \mathrm{~min}$ at room temperature to remove cells and other debris in the plasma. The supernatants were then centrifuged at $100,000 \times g$ for $30 \mathrm{~min}$ at $4^{\circ} \mathrm{C}$ to remove microvesicles that were larger than exosomes, harvested, and again centrifuged at $10,000 \times g$ for $70 \mathrm{~min}$ at $4^{\circ} \mathrm{C}$. Subsequently, the supernatants were gently decanted, and the exosome sediments were re-suspended in phosphate-buffered saline (PBS).

\section{Transmission electron microscopy}

The exosome suspension was diluted to $0.5 \mathrm{mg} / \mathrm{mL}$ with PBS, and then spotted onto a glow-discharged copper grid placed on a filter paper and dried for $10 \mathrm{~min}$ by exposure to infrared light. Next, the exosome samples were stained with one drop of phosphotungstic acid ( $1 \%$ aqueous solution) for $5 \mathrm{~min}$ and dried for 20 min by exposure to infrared light. Finally, the exosomes were visualized under a transmission electron microscope (HT7700, Hitachi, Tokyo, Japan) at $100 \mathrm{keV}$.

\section{Western blotting}

Previous studies have confirmed that Tsg101, CD63, and Alix are exosome biomarkers [25]. Western blotting was performed to assess the levels of these markers. Total proteins were extracted from exosome samples using lysis buffer. Each sample $(40 \mu \mathrm{g})$ was loaded onto a $12 \%$ sodium dodecyl sulfate-polyacrylamide gel and then transferred to a polyvinylidene fluoride membrane (Roche, Mannheim, Germany). These membranes were immersed in $2 \%$ bovine serum albumin at room temperature for $1 \mathrm{~h}$ and incubated with the following primary antibodies: anti-Tsg101 (1:1, 000, Santa Cruz Biotechnology, Santa Cruz, CA), anti-CD63 (1:2, 000, Abcam, Cambridge, UK), and anti-Alix (1:1, 000, Santa Cruz Biotechnology), followed by subsequent incubation with appropriate secondary antibodies after washing with PBS. Lastly, protein bands were visualized by incubating with an electro-chemiluminescence reagent.

\section{Nanoparticle tracking analysis}

The size of exosomes was measured using a Nanosight NS 300 system (NanoSight Technology, Malvern, UK). Exosomes were re-suspended in PBS at a concentration of $5 \mu \mathrm{g} / \mathrm{mL}$ and further diluted 500-1000-fold. Samples were manually injected into the sample chamber at room temperature. Each sample was configured with a $488 \mathrm{~nm}$ laser and a high-sensitivity sCMOS camera setting of 13 with an acquisition time of $30 \mathrm{~s}$ and a detection threshold setting of 7 . At least 200 completed tracks were analyzed per video. Finally, the results were analyzed using nanoparticle tracking analysis software.

\section{Total RNA extraction}

Total RNA of exosomes was extracted from plasma using TRIzol LS reagent (Invitrogen, Carlsbad, CA) according to the manufacturer's instructions and quantified using a NanoDrop ND-2000 spectrophotometer (Thermo Fisher Scientific, Wilmington, DE).

\section{Agilent methods for ceRNA array}

Total RNA from exosomes was isolated using TRIzol reagent (Invitrogen, Carlsbad, CA) according to the manufacturer's instructions and purified using a RNeasy Mini Kit (Qiagen GmBH, Hilden, Germany). RNA samples of each group were then used to generate fluorescently labeled cRNA targets for the human ceRNA array V1.0 ( 4 × $180 \mathrm{~K}$; Shanghai Biotechnology Corporation, Shanghai, China). The labeled cRNA targets were 


\section{Cellular Physiology Cell Physiol Biochem 2018;51:2704-2715 \begin{tabular}{ll|l} 
and Biochemistry Published onIIne: 12 December 2018 & $\begin{array}{l}\text { (c) } 2018 \text { The Author(s). Published by S. Karger AG, Basel } \\
\text { www.karger.com/cpb }\end{array}$ \\
\hline
\end{tabular}}

Table 1. Primer sequences used

\begin{tabular}{lcc}
\hline ProbeName & Cis_Target_Gene & Description \\
\hline LNCV6_84003 & ANXA2R & annexin A2 receptor \\
LNCV6_98602 & DDT & D-dopachrome tautomerase \\
LNCV6_108266 & HSD17B12 & hydroxysteroid 17-beta dehydrogenase 12 \\
LNCV6_116109 & CFLAR & CASP8 and FADD like apoptosis regulator \\
LNCV6_98390 & CACNG7 & calcium voltage-gated channel auxiliary subunit gamma 7 \\
\hline
\end{tabular}

then hybridized with the slides. After hybridization, slides were scanned on the Agilent Microarray Scanner (Agilent Technologies, Inc., Santa Clara, CA). Data were extracted using Feature Extraction software 10.7 (Agilent Technologies, Inc.). Raw data were normalized by the quantile algorithm of the limma package in the R program. Microarray experiments were performed at Shanghai Biotechnology Corporation following the protocol of Agilent Technologies, Inc.

Ratios between CRC and normal subjects (NC) were calculated. Genes with a fold change of at least 2 were selected for further analysis. The selected parent genes of circular RNA were grouped into functional categories based on the Gene Ontology database (http://www.geneontology.org/) and functional pathways (Kyoto Encyclopedia of Genes and Genomes) were also analyzed using the online enrichment analysis tool of Shanghai Biotechnology Corporation.

\section{Reverse transcription}

cDNA was synthesized by reverse transcription using the PrimeScript RT reagent kit (Takara Bio Inc., Kusatsu, Japan) with random primers according to the manufacturer's protocol.

\section{Real-time quantitative reverse transcription-polymerase chain reaction}

Real-time quantitative reverse transcription-polymerase chain reaction (qRT-PCR) was performed using SYBR Premix Ex Taq II (Tli RNaseH Plus; Takara Bio Inc.) on a CFX96 Real-Time PCR Detection System (Bio-Rad, Hercules, CA). Divergent primers were synthesized by Sangon Biotech (Shanghai, China). Data were analyzed using the $2^{-\Delta \mathrm{Ct}}$ method. All results are expressed as the mean \pm standard deviation of three independent experiments. The primer sequences used were as follows (Table 1).

\section{Prediction of IncRNA-related target genes}

Differentially expressed lncRNAs were selected for target prediction. The genes transcribed within a $10 \mathrm{kbp}$ window upstream or downstream of IncRNAs were considered as cis-acting target genes. The transacting target genes were predicted using RNAplex software.

\section{Statistics analysis}

All statistical analyses were performed using SPSS 22.0 software for Windows (SPSS, Chicago, IL) and GraphPad Prism 5.0 (GraphPad Software, La Jolla, CA). Differences in the levels of each lncRNA between CRC patients and paired samples were assessed using the $t$-test for paired data. P values less than 0.05 (twosided) were considered statistically significant. In this study, * indicates $\mathrm{P}<0.05$, ** indicates $\mathrm{P}<0.01$, and $* * *$ indicates $\mathrm{P}<0.001$.

\section{Results}

\section{Characterization of plasma exosomes}

To determine whether the particles isolated from the plasma by ultracentrifugation were exosomes, we performed transmission electron microscopy and western blot analysis. The isolated particles exhibited bona fide characteristics of exosomes, namely, sphericalshaped morphology with an approximately $100 \mathrm{~nm}$ size (Fig. 1A), and were found to be enriched with the exosomal marker proteins Tsg101, CD63, and Alix, as shown by western blotting (Fig. 1B). 
Peripheral plasma exosomal IncRNA profile in CRC patients

To verify whether there is a difference in the expression of IncRNAs between CRC patients and healthy subjects, we extracted plasma exosomal RNA from 10 CRC patients and 10 healthyindividualsand analyzed itusing the ceRNA array mentioned above. The results showed that there was a significant difference in the expression of IncRNAs between the two groups (Fig. 2). The expression of 1705 IncRNAs was significantly different, with 626 lncRNAs up-regulated and 1079 downregulated. In order to select viable biomarkers, we selected the following six IncRNAs with the largest increase in expression for subsequent analysis: LNCV6_116109, LNCV6_98390, LNCV6_38772,

LNCV6_108266, LNCV6_84003, and LNCV6_98602. Their levels in the plasma of CRC patients were significantly higher than those in the plasma of negative control individuals $(\mathrm{P}<0.05$, Fig. $3)$. The P-value was same for all six lncRNAs $(\mathrm{P}<0.05)$.

Verification of the highly expressed IncRNAs in CRC patients

In order to analyze the difference in expression of the six selected IncRNAs between CRC patients and healthy subjects, we extracted RNA from another cohort that included 50 CRC patients and 50 healthy subjects. The expression levels of the selected IncRNAs were examined by qRTPCR. As shown in Fig. 3, our data verified that the expression level of these lncRNAs in CRC patients was significantly higher than that in healthy individuals. The expression of LNCV6_116109 was most significantly up-regulated (about 7-fold; $\mathrm{P}<0.001$, Fig. $4 \mathrm{~A}$ ), while that of LNCV6_84003 was least significantly up-regulated (3-fold; $\mathrm{P}<0.05$,

Fig. 4E). Our results suggested that these six lncRNAs can be considered as diagnostic biomarkers for CRC (Fig. 4A-F).

\section{Expression pattern of the selected IncRNAs in early-stage CRC}

To evaluate the potential role of the selected IncRNAs in early diagnosis of CRC, we further analyzed their expressed patterns in each stage of CRC. As is shown in Fig. 5A-F, the levels of all the six IncRNAs were significantly up-regulated in stage I/II CRC patients compared with those in NC individuals. Furthermore, the levels of these IncRNAs were altered in each stage of CRC. These data suggested that the selected plasma exosomal lncRNAs might serve as potential biomarkers for the early detection of CRC. 
Fig. 3. Plasma expression levels of IncRNAs in CRC patients. (A-F) Expression levels of the six selected IncRNAs in CRC patients $(\mathrm{n}=10)$ and NC individuals $(n=10)$ were determined by qRT-PCR assay. Each point represents the relative expression level of certain lncRNAs in one sample. The expression of all six lncRNAs was up-regulated in CRC patients compared with that in $\mathrm{NC}$ individuals.

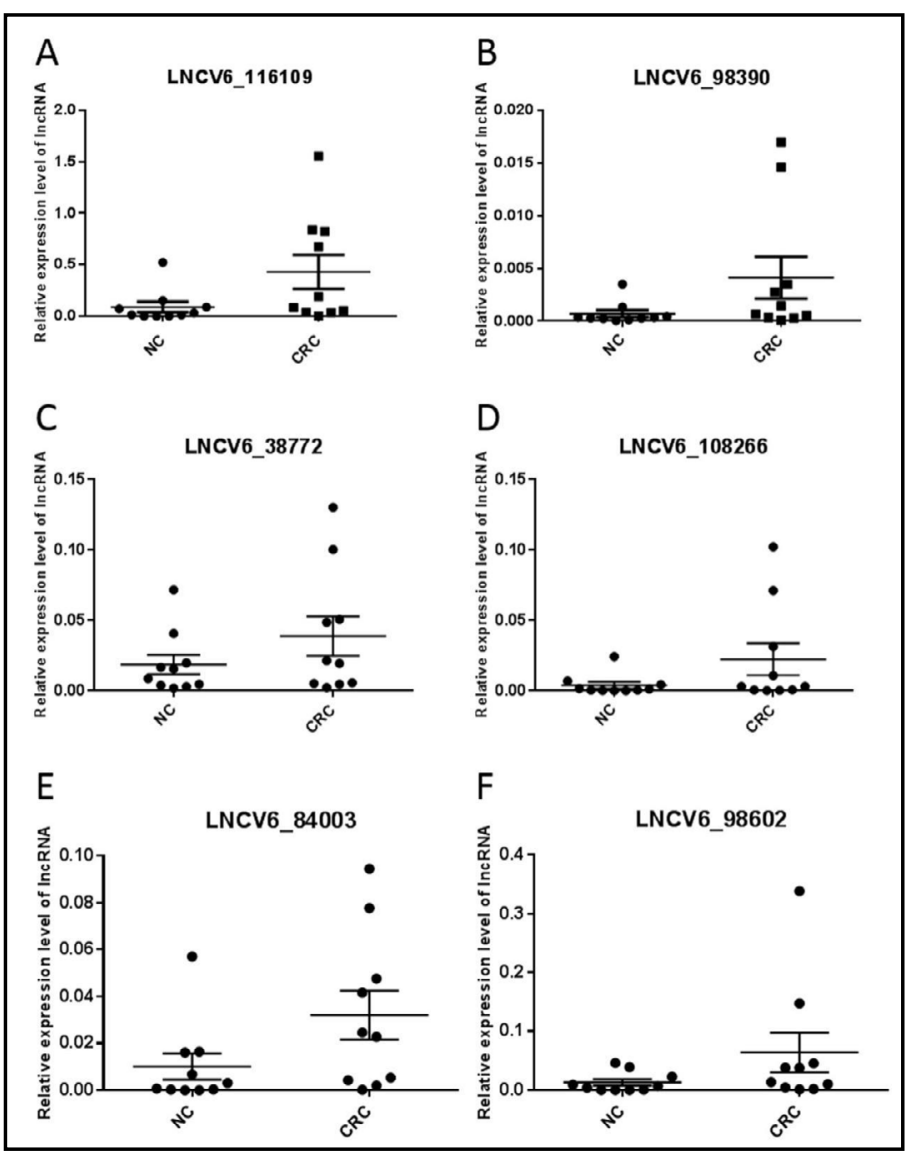

Fig. 4. Verification of the highly expressed lncRNAs in CRC patients. (A-F) Relative expression levels of each of the selected IncRNAs were verified in another cohort comprising CRC patients $(\mathrm{n}=50)$ and $\mathrm{NC}$ individuals $(\mathrm{n}=50)$ using qRT-PCR assay. * indicates $\mathrm{P}<0.05$, ** indicates $\mathrm{P}<0.01$, and ${ }^{* * *}$ indicates $\mathrm{P}<0.001$.

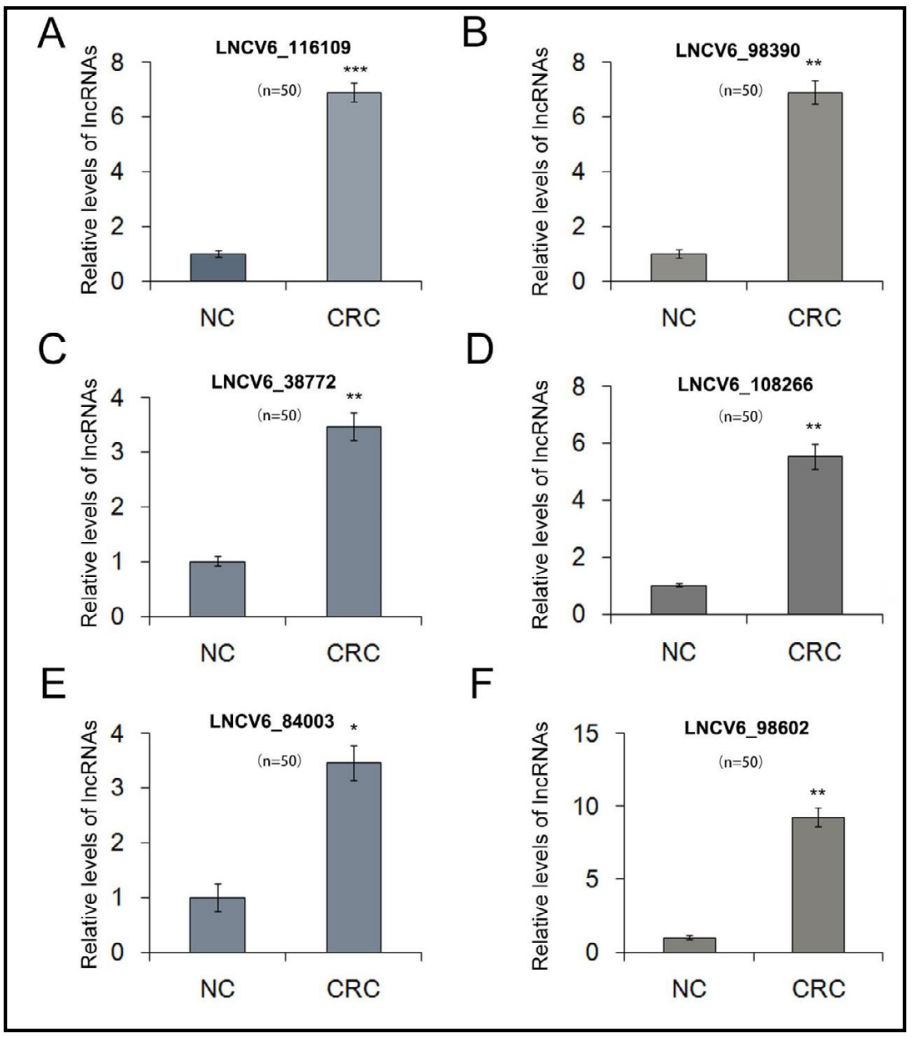


A

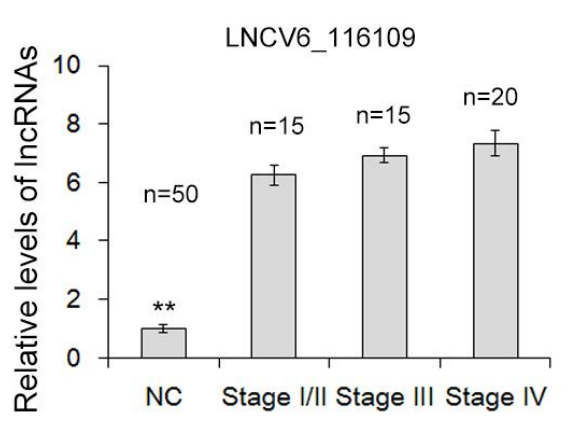

C

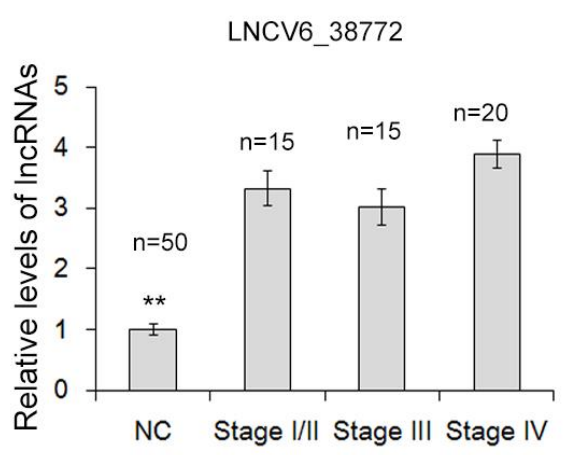

E

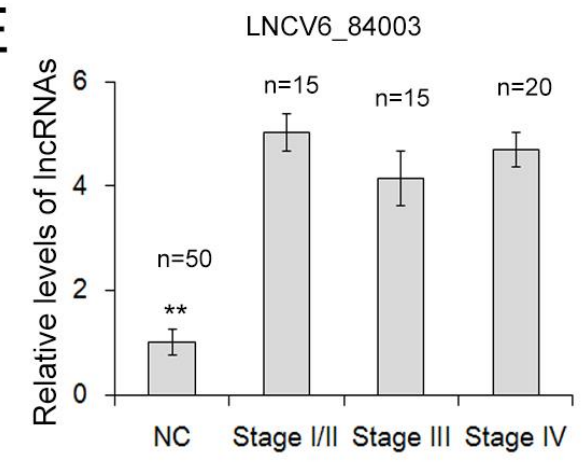

B

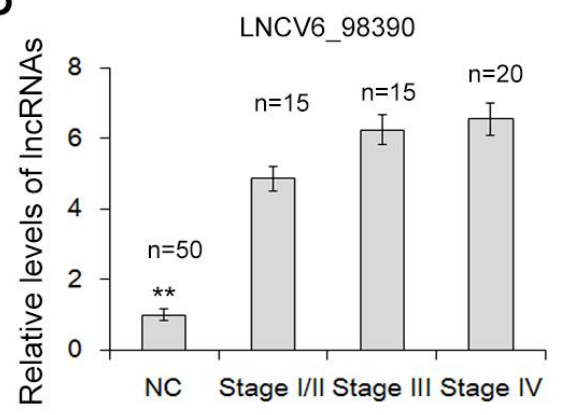

D

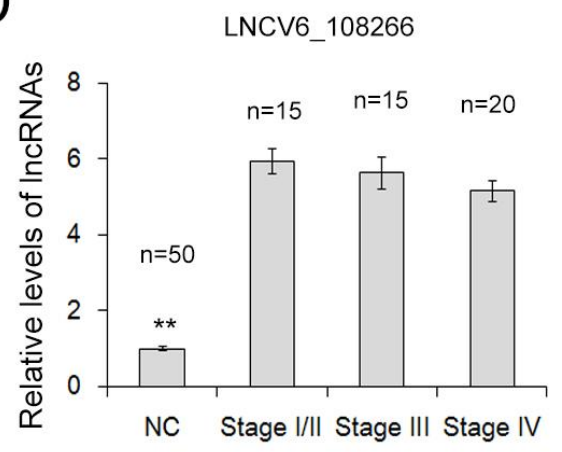

$\mathrm{F}$

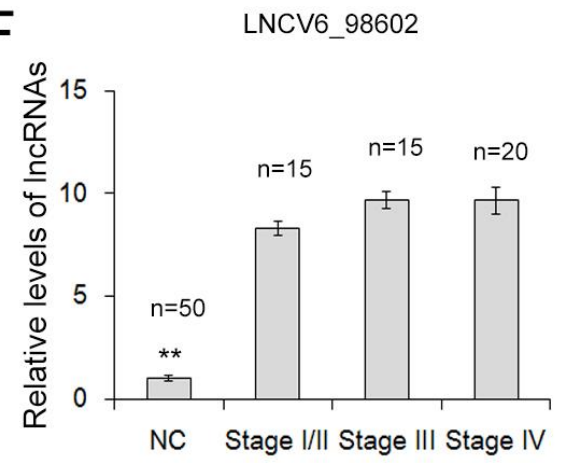

Fig. 5. Expression pattern of lncRNAs in each stage of CRC. Levels of the six selected lncRNAs were further analyzed in different clinical stages of CRC. Quantification of lncRNAs in healthy subjects $(\mathrm{n}=50)$ and patients with stage I/II CRC $(\mathrm{n}=15)$, stage III CRC $(\mathrm{n}=15)$, and stage IV CRC $(\mathrm{n}=20)$ is shown $(\mathrm{A}-\mathrm{F}) .{ }^{* *}$ indicates $\mathrm{P}<0.01$.

Potential diagnostic value of exosomal lncRNAs in CRC

Receiver operating characteristic (ROC) curves were then constructed to estimate the sensitivity and specificity of the six plasma exosomal lncRNAs. The values of the area under the curve (AUC) were 0.8052 (LNCV6_116109), 0.7088 (LNCV6_98390), 0.7460 (LNCV6_38772), 0.7292 (LNCV6_108266), 0.7356 (LNCV6_84003), and 0.6800 (LNCV6_98602) for the plasma samples (Fig. 6A-F).

Predicted downstream targets of IncRNAs

To further investigate the role of differentially expressed lncRNAs in CRC, we predicted their cis-target genes using bioinformatics methods. The target genes of LNCV6_84003, LNCV6_98602, LNCV6_108266, LNCV6_116109, LNCV6_98390, and LNCV6_38772 are shown in Table 2. 


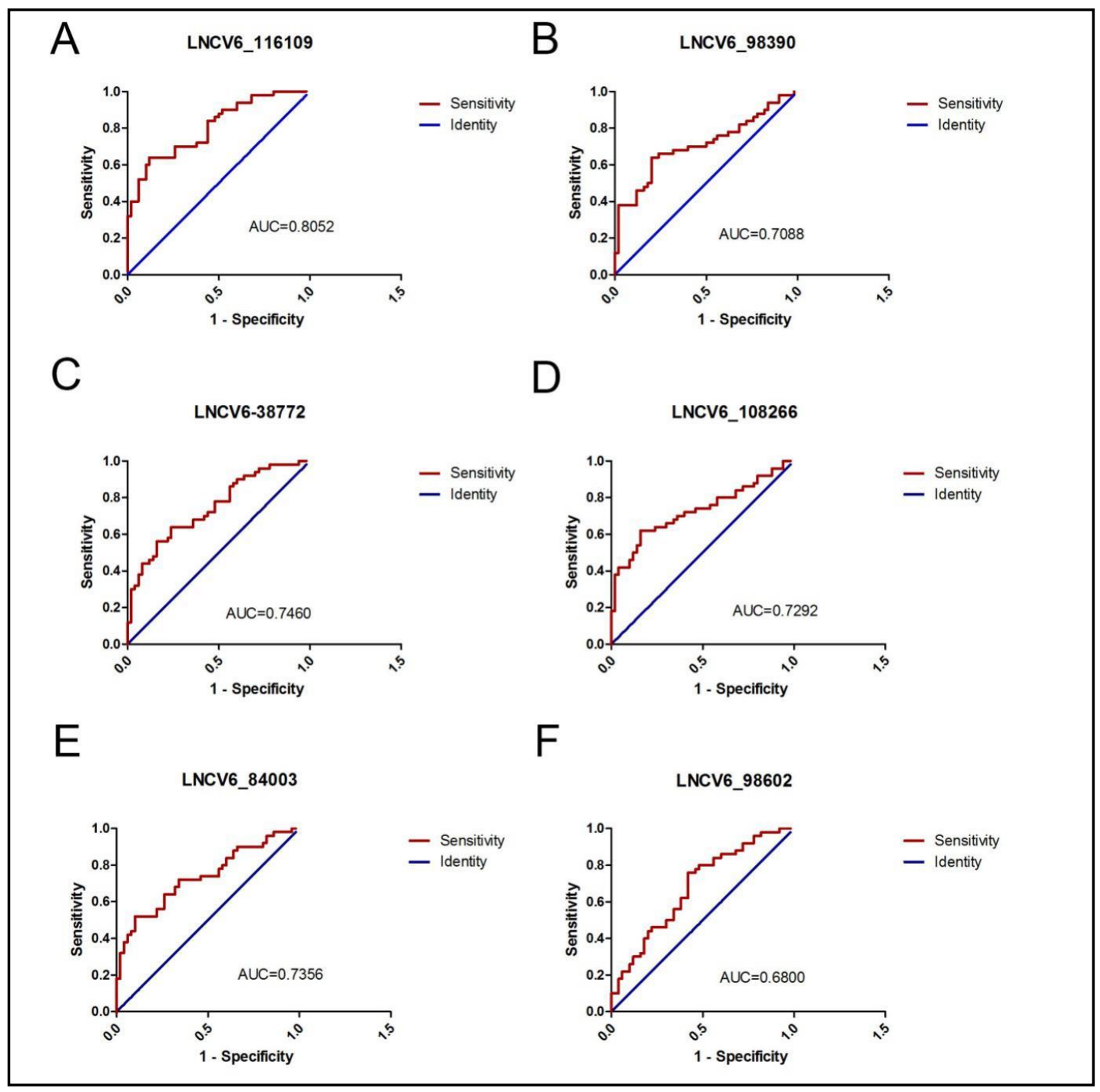

Fig. 6. ROC curve analysis of IncRNAs. (A-F) Graphs represent the AUC values. A. LNCV6_116109: AUC $=0.770$, 95\% confidence interval (CI) 0.5614-0.9786. B. LNCV6_98390: AUC $=0.7500$, 95\% CI 0.52860.9714. C. LNCV6_38772: AUC $=0.6500,95 \%$ CI 0.4031-0.8969. D. LNCV_108266: AUC $=0.6900,95 \%$ CI 0.4551-0.9249. E. LNCV6_84003: AUC $=0.7500,95 \%$ CI 0.5301-0.9699. F. LNCV6_98602: AUC $=0.7200$, 95\% CI 0.4926-0.9474.

\section{Discussion}

The survival of CRC patients is closely related to the stage at diagnosis. In the United States, patients with localized colon cancer have a $>90 \% 5$-year survival rate; however, this rate sharply decreases to $13 \%$ for patients diagnosed with distant metastasis [2]. Therefore, early diagnosis has become an urgent need for CRC prevention and
Table 2. The predicted Cis_targets of CRC-specific lncRNAs

\begin{tabular}{lc} 
Genes & \multicolumn{1}{c}{ Primers } \\
\hline LNCV6_116109 sense & GGGGACAGCAGTTCCAT \\
LNCV6_116109 anti-sense & ACAAGGAAAGCCTCCCA \\
LNCV6_98390 sense & TGTGGACGGTACATCTCCTC \\
LNCV6_98390 anti-sense & GGTCTAACCCCTGGGTCCTT \\
LNCV6_38772, sense & AGAAGAGTGTGTAGAAGGGA \\
LNCV6_38772, anti-sense & ATGAGCACCTCTTTGAATCC \\
LNCV6_108266, sense & TCAAAGTGGAGACAAGAACC \\
LNCV6_108266, anti-sense & CCAGCCCAACCTAAGATGC \\
LNCV6_84003, sense & CTAGCAGTTGACACAAACAG \\
LNCV6_84003, anti-sense & CCATTTCACAGATCAAGGGT \\
LNCV6_98602, sense & CTCGAGGACAAGTTCCTG \\
LNCV6_98602, anti-sense & AGGTTCTACTGGAGGCAG \\
\hline
\end{tabular}


treatment.

In recent years, with the use of FOBT and colonoscopy, the detection rate of CRC has improved. FOBT is easy to operate and inexpensive, but is limited by low sensitivity. Tumors tend to bleed, but not always, which may induce false-negative FOBT results [2628]. Colonoscopy is generally considered to be the gold standard for colorectal neoplasia [29]. However, this procedure is traumatic and painfulc with a prohibitive cost, making it unsuitable for large-scale screening [30]. Blood tests, such as tests for CEA, CA199, and CA242, have a low sensitivity, which does not meet the clinical diagnostic requirements [31].

Studies have shown that circulating RNAs are detected in the plasma or serum of cancer patients, making them novel biomarkers for tumor diagnosis [32].

Exosomes are drawing an increasing amount of attention for their specific characteristics. Studies have found that exosomes are secreted by various cell types and are involved in many biological functions under normal and pathological conditions [33, 34]. They can also serve as carriers of bioactive molecules to promote tumorigenesis [35]. In plasma, lncRNAs packaged into exosomes can be protected against degradation, thereby increasing their stability in circulation. For a stable RNA concentration, uncentrifuged clotted blood should be stored at $4^{\circ} \mathrm{C}$ and processed within $6 \mathrm{~h}$; long-time storage may cause its degradation [36].

Recently, many CRC-associated lncRNAs have been identified. Hu et al. reported that three dysregulated IncRNAs (AK123657, BX648207, and BX649059) are required for efficient invasion and proliferation suppression in CRC cell lines, which may provide an efficient classification tool for clinical prognosis evaluation of CRC [37]. Zheng et al. evaluated metastasis associated lung adenocarcinoma transcript 1 (MALAT-1) expression in 146 stage II/III CRC patients and 23 paired normal samples, and confirmed that its expression was up-regulated in CRC tissues, thereby suggesting that MALAT- 1 might serve as a negative prognostic marker [38]. Colon cancer-associated transcript family, HOX transcript antisense RNA, H19, and other IncRNAs such as plasmacytoma variant translocation 1 have been reported to be associated with CRC development [22]. Xia et al. reported 15 lncRNAs that were demonstrated to be linked with diverse biological processes in CRC progression [39]. These results were obtained by comparing tumor tissues and adjacent tissues, but the expression level of IncRNAs in these tissues was not necessarily the same as that in plasma [40]. Only a few studies have focused on the role of plasma lncRNAs in CRC diagnosis, and even fewer have focused on plasma exosomes. Wang et al. successfully established a distinctive serum 4-IncRNA panel (BANCR, NR_026817, NR_029373, and NR_034119) for CRC detection through stringent step-by-step selection procedures [41]. However, these stringent step-by-step procedures are not suitable for large sample screening. Our study is the first to link exosomal lncRNAs with CRC diagnosis.

In our study, we found that the expression level of exosomal lncRNAs was significantly up-regulated in CRC patients compared with that in healthy individuals. Among these, six highly expressed IncRNAs (LNCV6_116109, LNCV6_98390, LNCV6_38772, LNCV_108266, LNCV6_84003, and LNCV6_98602) were subjected to further verification. Results indicated that their plasma expression level in exosomes was up-regulated in CRC patients compared with that in normal subjects. ROC curve analysis confirmed the diagnostic values of these IncRNAs, suggesting their potential to be used as novel biomarkers for CRC.

Although we obtained positive results, further diverse studies are required to clarify the specific role of these lncRNAs in CRC; more cohorts should be analyzed in subsequent analyses. The stage of tumorigenesis at which the lncRNA expression level is up-regulated should also be investigated. Colorectal adenoma is considered the common benign precursor lesion for CRC transformation. Further studies should be performed to determine whether the expression level of these lncRNAs has already been altered at the very early stage of CRC. 


\section{Cellular Physiology Cell Physiol Biochem 2018;51:2704-2715 \begin{tabular}{ll|l} 
and Biochemistry & Dublished onlIne: 12 December 2018 & $\begin{array}{l}\text { ( } 2018 \text { The Author(s). Published by S. Karger AG, Basel } \\
\text { www.karger.com/cpb }\end{array}$ \\
\hline
\end{tabular}}

Hu et al.: Exosome IncRNAs are Biomarkers for CRC

\section{Conclusion}

In conclusion, by comparing the expression level of lncRNAs between different plasma exosomal samples, we identified a panel of six lncRNAs with high sensitivity and specificity that can be used as biomarkers for non-invasive screening of CRC.

\section{Acknowledgements}

This work was supported by grants from the National Natural Science Foundation of China (Nos. 81602158, 81802363). The funders had no role in study design; in collection, analysis, and interpretation of data; in the writing of the report; and in the decision to submit this article for publication. Dongzhi Hu and Yang Zhan performed most of the experiments, analyzed data, and wrote the manuscript. Ming Bai, Yiran Si and Jiayi Han reviewed and edited the manuscript and performed some experiments. Dalu Kong and Haiyang Zhang designed the experiments and edited the manuscript. Dalu Kong is the guarantor of this work and, as had full access to all of the data in the study and takes responsibility for the integrity of the data and the accuracy of the data analysis.

\section{Disclosure Statement}

The authors have no conflicts of interest to declare.

\section{References}

$>1$ Torre LA, Bray F, Siegel RL, Ferlay J, Lortet-Tieulent J, Jemal A: Global cancer statistics, 2012. CA Cancer J Clin 2015;65:87-108.

-2 Siegel RL, Miller KD, Fedewa SA, Ahnen DJ, Meester RGS, Barzi A, Jemal A: Colorectal cancer statistics, 2017. CA Cancer J Clin 2017;67:177-193.

-3 Maffei F, Zolezzi Moraga JM, Angelini S, Zenesini C, Musti M, Festi D, Cantelli-Forti G, Hrelia P: Micronucleus frequency in human peripheral blood lymphocytes as a biomarker for the early detection of colorectal cancer risk. Mutagenesis 2014;29:221-225.

4 Raposo G, Stoorvogel W: Extracellular vesicles: exosomes, microvesicles, and friends. J Cell Biol 2013;200:373-383.

5 Mateescu B, Kowal EJ, van Balkom BW, Bartel S, Bhattacharyya SN, Buzas EI, Buck AH, de Candia P, Chow FW, Das S, Driedonks TA, Fernandez-Messina L, Haderk F, Hill AF, Jones JC, Van Keuren-Jensen KR, Lai CP, Lasser C, Liegro ID, Lunavat TR, Lorenowicz MJ, Maas SL, Mager I, Mittelbrunn M, Momma S, Mukherjee K, Nawaz M, Pegtel DM, Pfaffl MW, Schiffelers RM, Tahara H, Thery C, Tosar JP, Wauben MH, Witwer KW, Nolte't Hoen EN: Obstacles and opportunities in the functional analysis of extracellular vesicle RNA - an ISEV position paper. J Extracell Vesicles 2017;6:1286095.

-6 Coumans FAW, Brisson AR, Buzas EI, Dignat-George F, Drees EEE, El-Andaloussi S, Emanueli C, Gasecka A, Hendrix A, Hill AF, Lacroix R, Lee Y, van Leeuwen TG, Mackman N, Mager I, Nolan JP, van der Pol E, Pegtel DM, Sahoo S, Siljander PRM, Sturk G, de Wever O, Nieuwland R: Methodological Guidelines to Study Extracellular Vesicles. Circ Res 2017;120:1632-1648.

7 Kourembanas S: Exosomes: vehicles of intercellular signaling, biomarkers, and vectors of cell therapy. Annu Rev Physiol 2015;77:13-27.

8 Ramirez MI, Amorim MG, Gadelha C, Milic I, Welsh JA, Freitas VM, Nawaz M, Akbar N, Couch Y, Makin L, Cooke F, Vettore AL, Batista PX, Freezor R, Pezuk JA, Rosa-Fernandes L, Carreira ACO, Devitt A, Jacobs L, Silva IT, Coakley G, Nunes DN, Carter D, Palmisano G, Dias-Neto E: Technical challenges of working with extracellular vesicles. Nanoscale 2018;10:881-906. 


\section{Cellular Physiology Cell Physiol Biochem 2018;51:2704-2715 and Biochemistry \begin{tabular}{l|l} 
DOI: 10.1159/000495961 2018 The Author(s). Published by S. Karger AG, Basel \\
(o) 2018
\end{tabular}

9 Nawaz M, Camussi G, Valadi H, Nazarenko I, Ekstrom K, Wang XQ Principe S, Shah N, Ashraf NM, Fatima F, Neder L, Kislinger T: The emerging role of extracellular vesicles as biomarkers for urogenital cancers. Nature Reviews Urology 2014;11:688-701.

10 Wang KC, Chang HY: Molecular mechanisms of long noncoding RNAs. Mol Cell 2011;43:904-914.

11 Zhang Y, Yang L, Chen LL: Life without A tail: new formats of long noncoding RNAs. Int J Biochem Cell Biol 2014;54:338-349.

12 Brannan CI, Dees EC, Ingram RS, Tilghman SM: The product of the H19 gene may function as an RNA. Mol Cell Biol 1990;10:28-36.

13 Walsh AL, Tuzova AV, Bolton EM, Lynch TH, Perry AS: Long noncoding RNAs and prostate carcinogenesis: the missing 'linc'? Trends Mol Med 2014;20:428-436.

14 Martianov I, Ramadass A, Serra Barros A, Chow N, Akoulitchev A: Repression of the human dihydrofolate reductase gene by a non-coding interfering transcript. Nature 2007;445:666-670.

15 Uhler JP, Hertel C, Svejstrup JQ: A role for noncoding transcription in activation of the yeast PHO5 gene. Proc Natl Acad Sci U S A 2007;104:8011-8016.

16 Martens JA, Laprade L, Winston F: Intergenic transcription is required to repress the Saccharomyces cerevisiae SER3 gene. Nature 2004;429:571-574.

17 Wu Z, Liu X, Liu L, Deng H, Zhang J, Xu Q, Cen B, Ji A: Regulation of IncRNA expression. Cell Mol Biol Lett 2014;19:561-575.

18 Martens-Uzunova ES, Bottcher R, Croce CM, Jenster G, Visakorpi T, Calin GA: Long noncoding RNA in prostate, bladder, and kidney cancer. Eur Urol 2014;65:1140-1151.

19 Xing Z, Park PK, Lin C, Yang L: LncRNA BCAR4 wires up signaling transduction in breast cancer. RNA Biol 2015;12:681-689.

20 Liu L, Zhang Y, Cao W: Highly expressed IncRNA LOC730101 promotes lung cancer cell growth through Wnt canonical pathway. Biochem Biophys Res Commun 2017;10.1016/j.bbrc.2017.09.104

-21 Fatima F, Nawaz M: Vesiculated Long Non-Coding RNAs: Offshore Packages Deciphering Trans-Regulation between Cells, Cancer Progression and Resistance to Therapies. Noncoding RNA 2017;3:

-22 Han D, Wang M, Ma N, Xu Y, Jiang Y, Gao X: Long noncoding RNAs: novel players in colorectal cancer. Cancer Lett 2015;361:13-21.

23 Kita Y, Yonemori K, Osako Y, Baba K, Mori S, Maemura K, Natsugoe S: Noncoding RNA and colorectal cancer: its epigenetic role. J Hum Genet 2017;62:41-47.

24 Momen-Heravi F, Balaj L, Alian S, Mantel PY, Halleck AE, Trachtenberg AJ, Soria CE, Oquin S, Bonebreak CM, Saracoglu E, Skog J, Kuo WP: Current methods for the isolation of extracellular vesicles. Biol Chem 2013;394:1253-1262.

25 Kumar D, Gupta D, Shankar S, Srivastava RK: Biomolecular characterization of exosomes released from cancer stem cells: Possible implications for biomarker and treatment of cancer. Oncotarget 2015;6:32803291.

26 Health Quality 0: Fecal occult blood test for colorectal cancer screening: an evidence-based analysis. Ont Health Technol Assess Ser 2009;9:1-40.

-27 Duffy MJ, van Rossum LG, van Turenhout ST, Malminiemi O, Sturgeon C, Lamerz R, Nicolini A, Haglund C, Holubec L, Fraser CG, Halloran SP: Use of faecal markers in screening for colorectal neoplasia: a European group on tumor markers position paper. Int J Cancer 2011;128:3-11.

28 Robertson DJ, Imperiale TF: Stool Testing for Colorectal Cancer Screening. Gastroenterology 2015;149:1286-1293.

29 Schreuders EH, Ruco A, Rabeneck L, Schoen RE, Sung JJ, Young GP, Kuipers EJ: Colorectal cancer screening: a global overview of existing programmes. Gut 2015;64:1637-1649.

30 Mandel JS: Screening for colorectal cancer. Gastroenterol Clin North Am 2008;37:97-115, vii.

31 Zhong W, Yu Z, Zhan J, Yu T, Lin Y, Xia ZS, Yuan YH, Chen QK: Association of serum levels of CEA, CA199, CA125, CYFRA21-1 and CA72-4 and disease characteristics in colorectal cancer. Pathol Oncol Res 2015;21:83-95.

-32 Schwarzenbach H, Hoon DS, Pantel K: Cell-free nucleic acids as biomarkers in cancer patients. Nat Rev Cancer 2011;11:426-437.

-33 Colombo M, Raposo G, Thery C: Biogenesis, secretion, and intercellular interactions of exosomes and other extracellular vesicles. Annu Rev Cell Dev Biol 2014;30:255-289. 


\section{Cellular Physiology Cell Physiol Biochem 2018;51:2704-2715

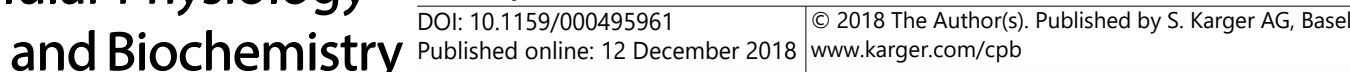

-34 Fatima F, Nawaz M: Long Distance Metabolic Regulation through Adipose-Derived Circulating Exosomal miRNAs: A Trail for RNA-Based Therapies? Frontiers in Physiology 2017;8:

35 Tkach M, Thery C: Communication by Extracellular Vesicles: Where We Are and Where We Need to Go. Cell 2016;164:1226-1232.

-36 Tsui NB, Ng EK, Lo YM: Stability of endogenous and added RNA in blood specimens, serum, and plasma. Clin Chem 2002;48:1647-1653.

-37 Hu Y, Chen HY, Yu CY, Xu J, Wang JL, Qian J, Zhang X, Fang JY: A long non-coding RNA signature to improve prognosis prediction of colorectal cancer. Oncotarget 2014;5:2230-2242.

-38 Zheng HT, Shi DB, Wang YW, Li XX, Xu Y, Tripathi P, Gu WL, Cai GX, Cai SJ: High expression of IncRNA MALAT1 suggests a biomarker of poor prognosis in colorectal cancer. Int J Clin Exp Pathol 2014;7:31743181.

39 Xie X, Tang B, Xiao YF, Xie R, Li BS, Dong H, Zhou JY, Yang SM: Long non-coding RNAs in colorectal cancer. Oncotarget 2016;7:5226-5239.

-40 Tong YS, Wang XW, Zhou XL, Liu ZH, Yang TX, Shi WH, Xie HW, Lv J, Wu QQ, Cao XF: Identification of the long non-coding RNA POU3F3 in plasma as a novel biomarker for diagnosis of esophageal squamous cell carcinoma. Mol Cancer 2015;14:3.

41 Wang R, Du L, Yang X, Jiang X, Duan W, Yan S, Xie Y, Zhu Y, Wang Q, Wang L, Yang Y, Wang C: Identification of long noncoding RNAs as potential novel diagnosis and prognosis biomarkers in colorectal cancer. J Cancer Res Clin Oncol 2016;142:2291-2301. 